\title{
ALGEBRAIC CORRECTION FOR METAL ARTIFACT REDUCTION IN COMPUTED TOMOGRAPHY
}

\author{
KIWAN JEON ${ }^{1}$, SUNG-HO KANG $^{1}$, CHI YOUNG AHN ${ }^{1}$, AND SUNGWHAN KIM ${ }^{2, \dagger}$ \\ ${ }^{1}$ Division of Computational Sciences in Mathematics, National Institute for Mathemati- \\ CAL SCIENCES, DAEJAON 305-811, KoREA \\ E-mail address: $\{$ jeonkiwan, runits, chiyoung\}@nims.re.kr \\ ${ }^{2}$ Division of Liberal Arts, Hanbat National University, DaEJaOn 305-719, Korea \\ E-mail address: sungwhan@hanbat.ac.kr
}

\begin{abstract}
If there are metals located in the X-ray scanned object, a point outside the metals has its range of projection angle at which projections passing through the point are disturbed by the metals. Roughly speaking, this implies that attenuation information at the point is missing in the blocked projection range. So conventional projection completion MAR algorithms to use the undisturbed projection data on the boundary of the metaltrace is less efficient in reconstructing the attenuation coefficient in detailed parts, in particular, near the metal region. In order to overcome this problem, we propose the algebraic correction technique (ACT) to utilize a prereconstructed interim image of the attenuation coefficient outside the metal region which is obtained by solving a linear system designed to reduce computational costs. The reconstructed interim image of the attenuation coefficient is used as prior information for MAR. Numerical simulations support that the proposed correction technique shows better performance than conventional inpainting techniques such as the total variation and the harmonic inpainting.
\end{abstract}

\section{INTRODUCTION}

Computed Tomography (CT) is an imaging modality for providing 2-D and 3-D highresolution tomographic images of the scanned human body from measured X-ray projections. Despite the controversy about the hazards associated with exposure to X-ray radiation, CT is a powerful tool to investigate the interior of the human body, and has been widely used for diagnostic and therapeutic purposes in various medical disciplines for decades. CT can provide high-resolution anatomic images in the absence of metallic objects such as dental fillings or prosthesis in the scanning path, but on the other hand metallic objects implanted in the human body cause severe metal streak artifacts in CT images and deteriorate reconstructed CT image quality. Usually metals are strongly attenuating objects and the detectors sensing X-ray

Received by the editors April 25 2014; Accepted May 27 2014; Published online May 302014.

2010 Mathematics Subject Classification. 44A12, 31A25, 68U10.

Key words and phrases. Computed Tomography, Metal Artifact Reduction, Algebraic Reconstruction Technique, Inpainting.

${ }^{\dagger}$ Corresponding author. 
beam attenuated by metals undergo severe photon starvation and thus after-log projection data become inaccurate.

Metal Artifact Reduction (MAR) to improve CT image quality is a hot issue of CT applications in clinical practice and several MAR algorithms have been proposed over past three decades. Lewitt and Bates first developed a MAR image reconstruction method from incomplete projections, in which the projection measurements through metals were assumed to be missing and were recovered by polynomial interpolation [1]. Besides linear and polynomial interpolations [2-4], wavelet interpolation [5,6], sinogram inpainting [7, 8], and normalized MAR interpolation [9] techniques have been proposed in order to fill the missing projection data. These MAR algorithms can be classified into the projection or sinogram completion methods. During the last decade, iterative methods modeling the physics behind metal artifacts have established another class of MAR algorithms in which the noise $[10,11]$ and the beam hardening $[12,13]$ were modeled. Compared to projection completion methods, modelbased iterative methods are computationally intensive and have limitations in clinical applications [14]. Recently, the hybrid method combining the projection completion method with the iterative method has been proposed $[15,16]$.

In the projection completion method, the missing projections on the metal trace can be filled up by continuing the flow of the uncorrupted projections adjacent to the metal trace in the help of various inpainting algorithms such as interpolation and total variation. Inpainting is the image processing technique of reconstructing lost or deteriorated parts of images and videos. For more details, see $[17,18]$. These traditional methods to fill the gap of the missing projections with the uncorrupted projections on the boundary of the metal trace in the sinogram may distort the true attenuation coefficient outside metal objects.

Fig. 1 (a), (b) and (c) show a phantom model containing metals located on two white regions, its sinogram and the sinogram region corresponding to a range of angle whose projections passing through the red point, as an example, in Fig. 1 (a) are blocked by the metal region, respectively. The closer a point is to the metal region, the wider the range of angle, in which projections passing through the point are disturbed by metals, is. Therefore the traditional filling methods may cause inaccurate information to projections passing through the red spot. As a result, CT images reconstructed by the filtered back projection (FBP) method from this corrected sinogram may be less efficient to recover the details of the true image of the phantom model. The efficiency of the projection completion method has high dependence of the accuracy of synthesized data.

In this paper we propose a new MAR algorithm which is called the algebraic correction technique (ACT) using an interim image of the attenuation coefficient outside the metal region. The attenuation coefficient is usually calculated from the measured projection data by FBP and algebraic reconstruction technique(ART) basically proposed by Kaczmarz [19]. Since FBP cannot be applied to incomplete data, we adopt ART which is expressed by a linear system with relationship between the uncorrupted projections and image values on rectangular grid. Since the matrix of the linear system is quite large and severely under-determined, various iterative algorithms to solve the linear system have been developed at the cost of computational time and memory [19-23]. In ACT, we deal with those limitations and improve the imaging 


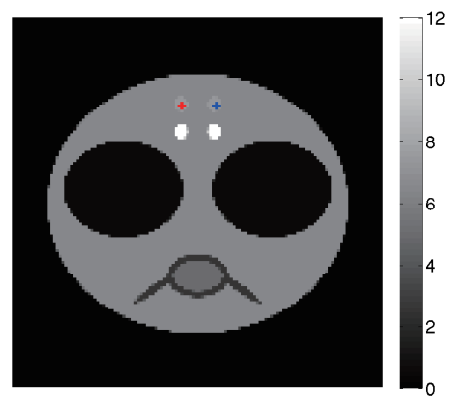

(a)

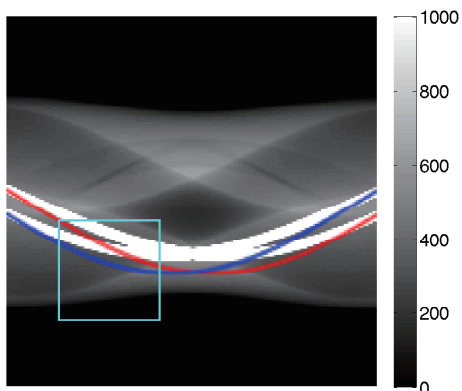

(b)

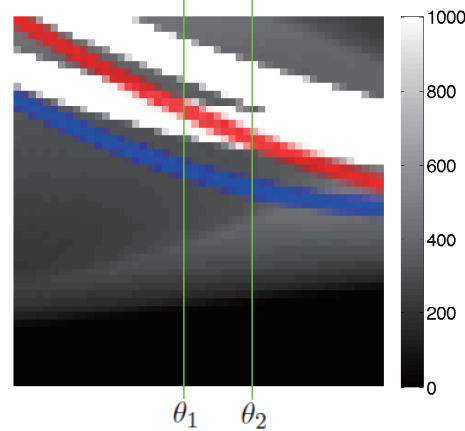

(c)

FIGURE 1. (a) shows a phantom model containing two metals which are located on white regions. (b) is its sinogram. Two blue and red curves indicate the traces of projections passing through blue and red spots in (a) located outside the metal regions. (c) shows the magnified image of the boxed region in (b). Projections passing through the red point are blocked by the metals in a range of projection angle. (For interpretation of the references to color in this figure, the reader is referred to the web version of this article.)

quality efficiently by generating a linear system at coarse scale that are solved in relatively short time by the least squares method equipped with Tikhonov regularization and reconstructing the corresponding low resolution interim image. The temporary image of the attenuation coefficient is exploited as prior information for MAR. Assuming that the attenuation value is identically zero on the metal region, we project the solution of the linear system into the metal trace and interpolate synthesized projections on the metal trace in order to replace the corrupted projection data. The final image of the attenuation coefficient is hence obtained from this surrogate sinogram by FBP.

This paper is organized as follows. In Section 2, we give a brief introduction to ARTs and explain the proposed algebraic correction method ACT. In Section 3, numerical results are provided to demonstrate ACT and we compare its performance with those of traditional inpainting techniques including the total variation and the harmonic inpainting. Finally we finish the paper with conclusion and future works.

\section{METHOD}

2.1. Algebraic Reconstruction Technique. Algebraic Reconstruction Technique (ART) can be traced to an iterative technique introduced by S. Kaczmarz [19] and was first applied in CT [20]. In ART, it is assumed that a certain reconstruction region, for instance rectangle or disk, is known and the region completely contains the scanned object. The region is then discretized with square grids and we obtain the following relationship between the measured projection data $\mathbf{p}=\left(p_{1}, p_{2}, \cdots, p_{M}\right) \in \mathbb{R}^{M}$ and the unknown image $\mathbf{f}=\left(f_{1}, f_{2}, \cdots, f_{N}\right) \in \mathbb{R}^{N}$ 


$$
\sum_{j=1}^{N} w_{i j} f_{j}=p_{i}, \quad i=1,2, \cdots, M
$$

where $M$ is the number of projections measurements, $N$ is the number of grids, and $w_{i j}$ is the weighting factor which is equal to the fractional length or area of the $j$-th image pixel intercepted by the $i$-th ray. Note that most of the $w_{i j}$ 's are zero since only a small number of image pixels contribute to any projection and the matrix $W=\left(w_{i j}\right)$ is sparse. An iterative algorithm to solve the linear system (2.1) was basically proposed by S. Kaczmarz [19] which can be expressed as

$$
\mathbf{f}^{(i)}=\mathbf{f}^{(i-1)}-\frac{<\mathbf{f}^{(i-1)}, \mathbf{w}_{i}>-p_{i}}{<\mathbf{w}_{i}, \mathbf{w}_{i}>} \mathbf{w}_{i}, \quad i=1,2, \cdots, M
$$

where $\left\langle\cdot, \cdot>\right.$ is the dot product in the space $\mathbb{R}^{N}, \mathbf{w}_{i}=\left(w_{i 1}, w_{i 2}, \cdots, w_{i N}\right) \in \mathbb{R}^{N}$, and $\mathbf{f}^{(i)}$ is the orthogonal projection of $\mathbf{f}^{(i-1)}$ onto the hyperplane $<\mathbf{f}, \mathbf{w}_{i}>=p_{i}$. The method (2.2) is based on considering the solution $\mathbf{f}$ as a point in $\mathbb{R}^{N}$ which is the intersection of $\mathrm{M}$ hyperplanes. The convergence of the above iterative algorithm was investigated by K. Tanabe [21].

However, since $N$ is often much bigger than $M$, the linear system (2.1) is severely underdetermined. More worse, the projections obtained in CT often contain noise which would cause $\mathbf{p}$ not to lie in the range of the operator W. Even if convergence is guaranteed, the method (2.2) would then converge very slowly to the solution. So many other iterative algorithms have been developed. Simultaneous Iterative Reconstructive Technique (SIRT, [22]) and Simultaneous Algebraic Reconstructive Technique (SART, [23]) are variations of the Kaczmarz's method.

2.2. Algebraic Correction Technique for MAR. Fig. 2 (a) shows a phantom model containing two metal regions implanted in white areas and several regions with different attenuation values. Fig. 2 (b) shows the sinogram of the phantom model. FBP generates an image with streak artifacts caused by a metal object as shown in Fig. 2 (c). Using a simple thresholding, we can determine the metal region in the reconstructed image. Then we cut off the corrupted projections from the sinogram whose rays lie on the metal region. With the help of conventional inpainting methods such as the total variation and harmonic inpainting methods, the missing projections can be filled up so that FBP provides an improved image without streak artifacts from the synthesized sinogram. Fig. 2 (d1) and (d2) are the sinogram filled by the harmonic inpainting method and its FBP image, respectively. Fig. 2 (e1) and (e2) are the sinogram filled by the total variation method and its FBP image, respectively.

Reconstructed images (d2) and (e2) are significantly improved compared with the reconstructed image (c). However, it is difficult to distinguish two small disc regions, which are originally located above the metal region, in (d2) and (e2). The reason is as follows. Let $\mathbf{r}_{0}$ be a point in the disc regions, for instance, the red spot in Fig. 1 (a). The red curve in Fig. 1 (b) traces the projections whose rays pass through the point $\mathbf{r}_{0}$. Fig. 1 (c), which is a magnified one of the boxed region in Fig. 1 (b), shows the blind part of attenuation information of the red point $\mathbf{r}_{0}$. In other words, the projection rays passing through the point $\mathbf{r}_{0}$ at projection angle 
from $\theta_{1}$ to $\theta_{2}$ are blocked by the metal objects and hence there is no projection containing attenuation information at $\mathbf{r}_{0}$ on the range of angle $\left[\theta_{1}, \theta_{2}\right]$. So conventional filling of the missing projections in the box using the neighboring uncorrupted data may ignore information on the attenuation value at the point $\mathbf{r}_{0}$, and hence CT images reconstructed by FBP from this manipulated sinogram are less efficient to recover the details of the true image of the attenuation coefficient of the phantom model.

In this paper, we take a different approach to filling up the metal trace in the sinogram. Instead of using the boundary projections of the metal trace, we exploit an interim image of the attenuation coefficient reconstructed from incomplete projection data. Let $M$ be the number of measured projection data for all projection angles and let $\mathbf{p}=\left(p_{1}, p_{2}, \cdots, p_{M}\right) \in \mathbb{R}^{M}$ be the measured projection data. We categorize the projections $p_{k}$ into two parts of corrupted and uncorrupted ones. Let $\mathbf{p}_{t}=\left(p_{t_{1}}, \cdots, p_{t_{m_{1}}}\right) \in \mathbb{R}^{m_{1}}$ be the vector of uncorrupted projections whose ray does not touch metal objects, and let $\mathbf{p}_{s}=\left(p_{s_{1}}, \cdots, p_{s_{m_{2}}}\right) \in \mathbb{R}^{m_{2}}$ be the vector of corrupted projections whose ray passes through metal objects. Note that $m_{1}+m_{2}=M$. In many clinical practices, the measurement number $M$ is huge. Even though the corrupted data $\mathbf{p}_{s}$ is eliminated from the whole data $\mathbf{p}, m_{1}$ is still so big that applying the method (2.2) encounters memory limitations and requires considerable computation time. So we use coarse square grids $D_{i}, i=1,2, \cdots, N$, with width $\delta$ bigger than the size of a detector and let $\mathbf{f}=\left(f_{1}, f_{2}, \cdots, f_{N}\right) \in \mathbb{R}^{N}$ be the image vector. If the area of the intersection of $D_{i}$ and the metal region is bigger than $\delta^{2} / 2$, we suppose that the image value $f_{i}$ is zero. Here there are several ways to generate linear systems (2.1) with various size, but we want the size of the generated linear system to be not big. So we propose to select $N$ uncorrupted projections $p_{t_{k}}$ from $\mathbf{p}_{t}$ so that they are evenly distributed through components of $\mathbf{p}_{t}$ in index order. Let $\tilde{\mathbf{p}}_{t} \in \mathbb{R}^{N}$ be a vector whose elements are the selected uncorrupted projections $p_{t_{k}}$. Then we have the matrix form of the linear system (2.1)

$$
W \mathbf{f}=\tilde{\mathbf{p}}_{t}
$$

Here, we emphasize that the weight matrix $W$ is not so big that we apply the Kaczmarz's iterative method (2.2) or its variations such as SIRT and SART. So in order to solve (2.3), we apply the well-known least squares method equipped with the Tikhonov regularization defined by

$$
\underset{\mathbf{f}}{\arg \min }\left(\left\|W \mathbf{f}-\tilde{\mathbf{p}}_{t}\right\|_{2}^{2}+\alpha\|\mathbf{f}\|_{2}^{2}\right)
$$

where $\alpha$ is the regularization parameter. Then (2.4) has the unique solution

$$
\mathbf{f}=\left(W^{T} W+\alpha I\right)^{-1} W^{T} \tilde{\mathbf{p}}_{t}
$$

where $I \in \mathbb{R}^{N \times N}$ is the identity matrix.

Here note that the solution $\mathbf{f}$ in (2.5) is a temporary image of the attenuation coefficient on coarse grids $D_{i}$. We synthesize sporadic projection data from the image $\mathbf{f}$ and interpolate the synthesized projection data into the metal trace in the sinogram in order to replace the corrupted data $\mathbf{p}_{s}$. Finally, we apply FBP in order to obtain the final reconstructed image from this updated sinogram. 


\section{RESULTS}

3.1. Summary of the proposed method. Unlike the conventional inpainting methods to use the boundary data of the metal trace in the sinogram, the proposed ACT exploits an interim image of the attenuation coefficient as prior information for MAR. Briefly, the proposed method is based on the following steps:

Step 1: Reconstruct an FBP image from the measured projection data $\mathbf{p .}$

Step 2: Using a simple thresholding, find the image of the metal region in the reconstructed image and cut off the metal trace from the sinogram.

Step 3: Decompose the vector $\mathbf{p}$ into two vectors $\mathbf{p}_{s}$ whose elements are projections corrupted by metal objects and $\mathbf{p}_{t}$ whose elements are uncorrupted projections outside the metal trace.

Step 4: Discretize the phantom with coarse square grids $D_{i}, i=1,2, \cdots, N$. Select evenly $N$ uncorrupted projections from $\mathbf{p}_{t}$ and let $\tilde{\mathbf{p}}_{t}$ be the vector of $N$ selected projections.

Step 5: Find a solution $\mathbf{f}$ defined in (2.5). If the area of the intersection of $D_{i}$ and the metal region is bigger than half of the area of $D_{i}$, we suppose that the image value $f_{i}$ is zero.

Step 6: Project the solution $\mathrm{f}$ into the metal trace.

Step 7: Interpolate projected projections of $\mathbf{f}$ on the metal trace in order to replace $\mathbf{p}_{s}$.

Step 8: Using FBP, reconstruct the final image from the updated sinogram.

3.2. Numerical Experiment. We use an attenuation coefficient distribution as depicted in Fig. 2 (a). The image size is $128 \times 128$. We set the infinitesimal rotation angle for the simulated projection to $1^{\circ}$. The sinogram hence has the size of $182 \times 180$ (Fig. 2 (b)). To obtain the projection image and perform the FBP in the procedure, we use an open source project, called scikit-image [24], running on the python. Also, in order to solve the minimization problem (2.4), we use the numpy [25] which is a famous linear algebra library on the python. For the acceleration of the linear solver in the numpy, we build the numpy with Intel MKL (math kernel libraries) that enables to use all core of the Intel CPU. We test all numerical experiments on 64-bit Ubuntu 12.04. LTS with Intel i7 CPU (3.4GHz, quad core) and 16GB memory.

Fig. 3 (a) shows the interim images obtained by solving the minimization problem (2.4) on the coarse grid. Clearly, we see that the reconstruction result is less affected by metal artefact. From the obtained images, we perform the forward projection to get a prior information for the 


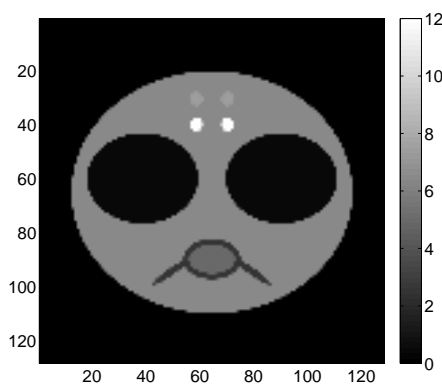

(a)

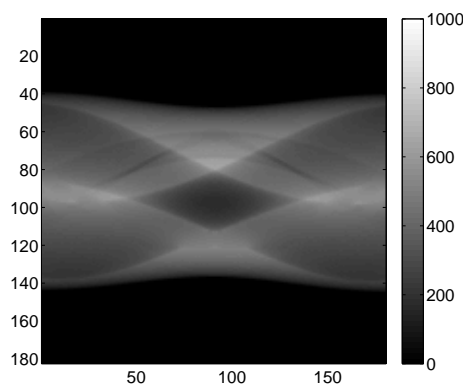

(d1)

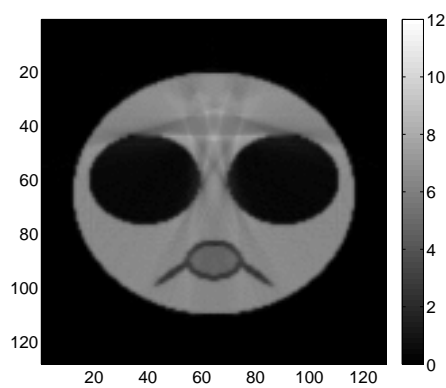

(d2)

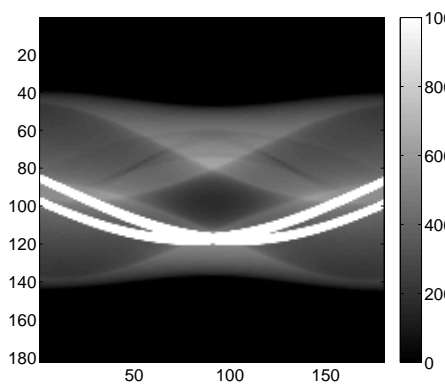

(b)

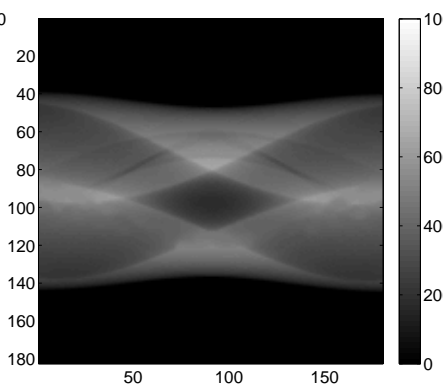

(e1)

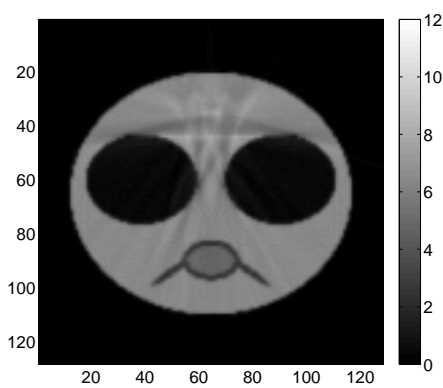

(e2)

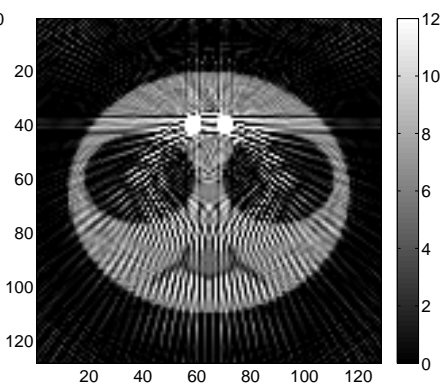

(c)

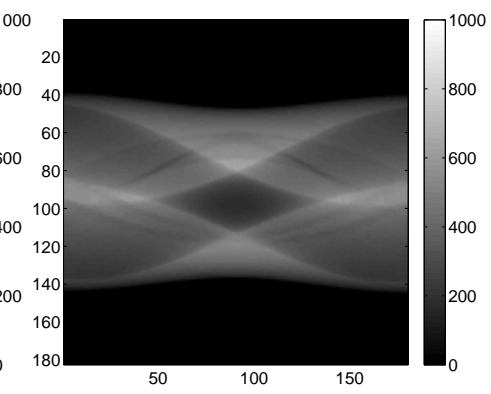

(f1)

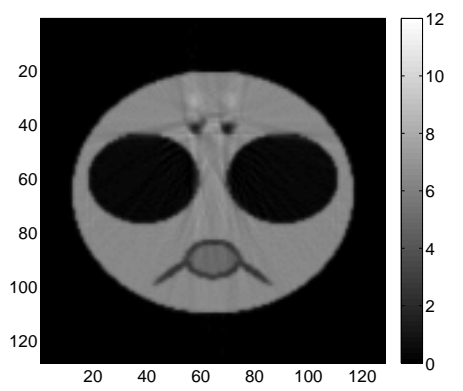

(f2)

FIGURE 2. (a) Original image containing metal regions. (b) Sinogram of (a). (c) FBP reconstructed image from the sinogram (b). (d1) Inpainted sinogram using the harmonic inpainting. (d2) FBP reconstructed image from the sinogram (d1). (e1) Inpainted sinogram using the total variation. (e2) FBP reconstructed image from the sinogram (e1). (f1) Sinogram using proposed correction method. (f2) FBP reconstruction image from the sinogram (f1). 


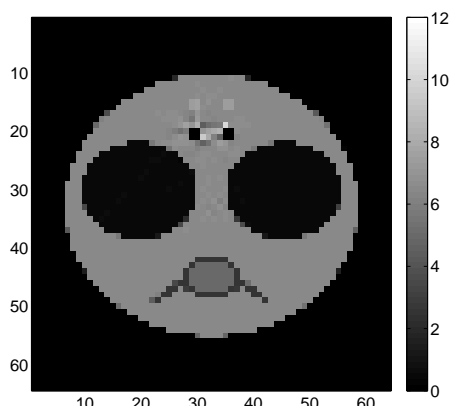

(a)

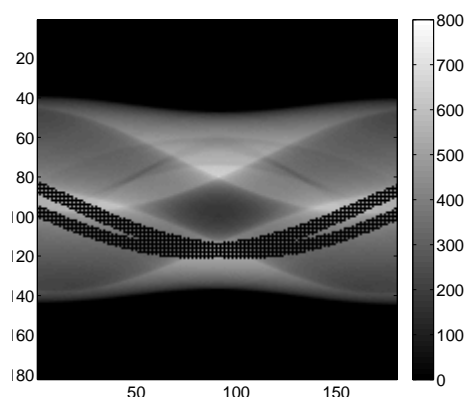

(c)

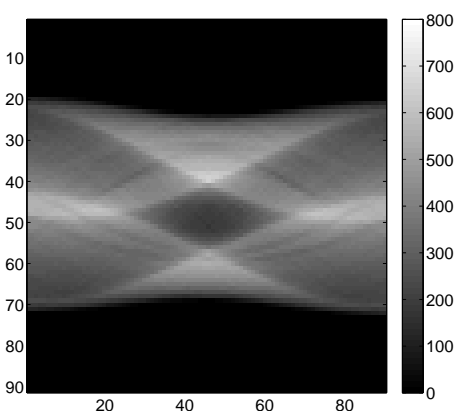

(b)

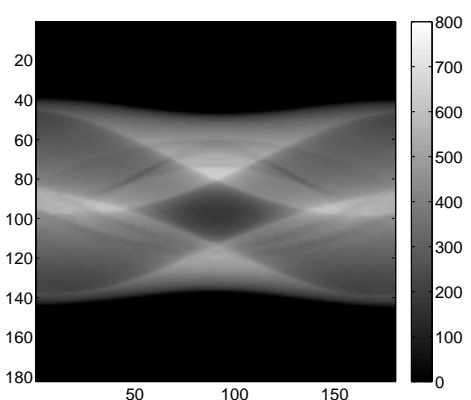

(d)

FIGURE 3. Description of correction procedure. (a) reconstructed image on coarse grid, (b) the sinogram of (a), (c) correction of sinogram on fine grid and (d) correction of the remaining points in the metal trace region using harmonic inpainting.

sinogram correction (See Fig. 3 (b)). Fig. 3 (c) presents the correction step using information that the fine grid is twice of coarse one in scale. We put the projection data of the coarse grid on even points of the fine grid in the metal trace region. Then, we apply the harmonic inpainting to fill the remaining points in the metal trace region (See Fig. 3 (d)).

Fig. 2 (f2) is the final result by applying FBP to Fig. 2 (f1).

To verify the numerical experiment, we illustrate the 1-D profiles of reconstructed images. Fig. 4 (a) illustrates zoomed-in images of the original, the proposed algorithm and the conventional harmonic inpainting from top to bottom, respectively. The gray, red and blue lines of Fig. 4 (b) mean the 1-D profile of the left images, respectively. Apparently, we see that the proposed method can reconstruct the small inclusion regions defected by metal artefact.

\section{Conclusion}

We propounded a question about efficiency of existing inpainting algorithms for MAR to use the boundary projections of the metal trace in the sinogram. In order to settle the limitation, 


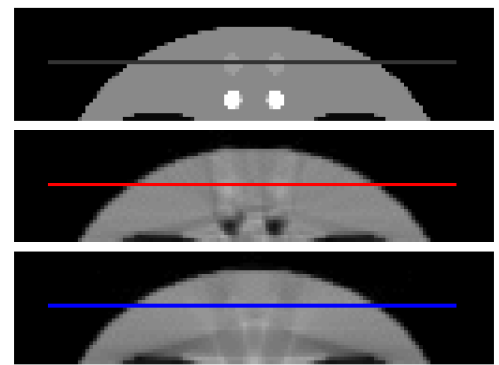

(a)

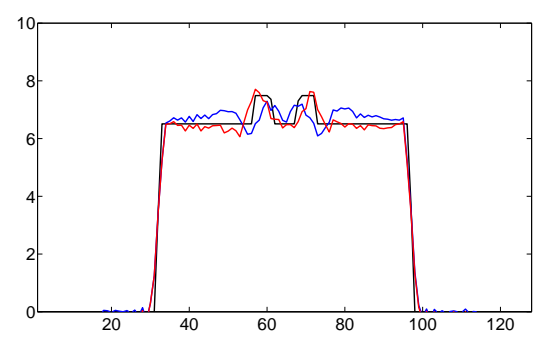

(b)

FIGURE 4. Performance comparison of the proposed ACT and the MAR harmonic inpainting algorithm through 1D profiles. (For interpretation of the references to color in this figure, the reader is referred to the web version of this article.)

we proposed the algebraic correction technique (ACT) to utilize an interim image of the attenuation coefficient outside the metal region which was obtained by solving a carefully designed linear system. We demonstrated numerically that ACT improves image quality in detailed parts compared with conventional inpainting methods such as the total variation and the harmonic inpainting. This suggests its usefulness in medical diagnosis and other medical applications.

In ACT, it is an essential part to find a temporary image of the attenuation coefficient from the incomplete projection data outside the metal trace. Using the temporary image, the missing projections on the metal trace are calculated. To do so, we are forced to solve a linear system with the incomplete projection data. In this paper, in the purpose of avoiding possible computational cost in dealing with a large linear system, we generated a linear system defined on coarse grids and solved it using the Tikhonov regularized least squares method. However, there is not a study on design of a linear system and its inversion method to minimize computational cost and maximize image quality. So future works should focus on optimization of ACT.

\section{ACKNOWLEDGMENTS}

This work was supported by the National Institute for Mathematical Sciences(NIMS) grants funded by the Korea government(No. A21402).

\section{REFERENCES}

[1] R.M. Lewitt and R.H.T. Bates, Image reconstruction from projections: III: Projection completion methods (theory), Optik, $\mathbf{5 0}$ (1978), 189-204.

[2] M. Abdoli, M.R. Ay, A. Ahmadian, and H. Zaidi, A virtual sinogram method to reduce dental metallic implant artefacts in computed tomography-based attenuation correction for PET, Nucl. Med. Commun.,31 (2010), $22-31$.

[3] G.H. Glover and N.J. Pelc, An algorithm for the reduction of metal clip artifacts in CT reconstructions, Med Phys, 8 (1981), 799-807. 
[4] W.A. Kalender, R. Hebel, and J. Ebersberger, Reduction of CT artifacts caused by metallic implants, Radiology, 164 (1987), 576-577.

[5] A. Mehranian, M.R. Ay, A. Rahmim, and H. Zaidi, X-ray CT Metal Artifact Reduction Using Wavelet Domain $L_{0}$ Sparse Regularization, IEEE Transactions on Medical Imaging, 32(2013), 1707-1722.

[6] S. Zhao, D.D. Robertson, G. Wang, B. Whiting, and K.T. Bae, X-ray CT metal artifact reduction using wavelets: An application for imaging total hip prostheses, IEEE Trans. Med. Imag., 19 (2000), 1238-1247.

[7] Y. Chen, Y. Li, H. Guo, Y. Hu, L. Luo, X. Yin, J. Gu, and C. Toumoulin, CT Metal Artifact Reduction Method Based on Improved Image Segmentation and Sinogram In-Painting, Mathematical Problems in Engineering, 2012, Article ID 786281

[8] H. Xue, L. Zhang, Y. Xiao, Z. Chen, and Y. Xing, Metal Artifact Reduction in Dual Energy CT by Sinogram Segmentation Based on Active Contour Model and TV Inpainting, IEEE Nuclear Science Symposium Conference Record, (2009), 904-908.

[9] E.Meyer, R. Raupach, M. Lell, B. Schmidt, and M. Kachelriess, Normalized metal artifact reduction (NMAR) in computed tomography, Med. Phys., 37 (2010), 5482-5493.

[10] B. De Man, J. Nuyts, P. Dupont et al., Reduction of metal streak artifacts in $x$-ray computed tomography using a transmission maximum a posteriori algorithm, IEEE Trans. Nucl. Sci., 47 (2000), 977-981.

[11] J. Nuyts, B. De Man, P. Dupont, et al., Iterative reconstruction for helical CT: a simulation study, Phys. Med. Biol., 43 (1998), 729-737.

[12] B. De Man, J. Nuyts, P. Dupont et al., An iterative maximum-likelihood polychromatic algorithm for CT, IEEE Trans. Med. Imaging, 20 (2001), 999-1008.

[13] C.H. Yan, R.T. Whalen, G.S. Beaupr et al., Reconstruction algorithm for polychromatic CT imaging: application to beam hardening correction, IEEE Trans. Med. Imaging, 19 (2000), 1-11.

[14] J. Choi, et al. Sparsity Driven Metal Part Reconstruction for Artifact Removal in Dental CT, Journal of X-ray Science and Technology, 19 (2011), 457-475.

[15] F.E. Boas and D. Fleischmann, Evaluation of two iterative techniques for reducing metal artifacts in computed tomography, Radiology, 259 (2011), 894-902.

[16] M. Oehler and T.M. Buzug, Statistical Image Reconstruction for Inconsistent CT Projection Data, Methods Inform. Med., 46 (2007), 261-269.

[17] M. Bertalmio, G. Sapiro, V. Caselles and C. Ballester, Image Inpainting, Proceedings of SIGGRAPH 2000, New Orleans, USA, July 2000.

[18] T. F. Chan and J. Shen, Mathematical Models for Local Nontexture Inpainting, SIAM J. Applied Math., 62 (2001), 1019-1043.

[19] S. Kaczmarz, Angentihrte Auflosung von Systemen linearer Gleichungen, Bull. Int. Acad. Pol. Sei. Lett., 35 (1937), 355-357.

[20] R. Gordon, R. Bender, and G.T. Herman, Algebraic reconstruction techniques (ART) for three-dimensional electron microscopy and X-ray photography, J. Theoret. Biol., 29 (1970), 471-482.

[21] K. Tanabe, Projection method for solving a singular system, Numer. Math., 17 (1971), 203-214.

[22] P. Gilbert, Iterative methods for the reconstruction of three dimensional objects from their projections, J. Theor. Biol., 36 (1972), 105-117.

[23] A.H. Andersen and A.C. Kak, Simultaneous algebraic reconstruction technique (SART): A superior implementation of the art algorithm, Ultrason. Imaging, 6 (1984), 81-94.

[24] S. Walt, J.L. Schönberger, J. N.-Iglesias, F. Boulogne, J.D. Warner et al., Scikit-image: image processing in python, http://scikit-image.org.

[25] E. Jones, T. Oliphant, P. Petersonet et al., SciPy: Open source scientific tools for Python, http://www.scipy.org. 